\title{
Le immagini del rimorso nella Cognizione del dolore di Gadda
}

\author{
Linda GAROSI \\ Universidad de Córdoba ${ }^{1}$ \\ linda.garosi@uco.es
}

Recibido: $25 / 06 / 2012$

Aceptado: $15 / 07 / 2012$

\section{RIASSUNTO}

Il presente articolo affronta l'analisi di un aspetto peculiare del romanzo La cognizione del dolore di Carlo Emilio Gadda. È nostro proposito mettere in evidenza il nesso di unione che lega le fotografie possedute dall'autore e alcune sequenze del romanzo. I ritratti fotografici, che preservano intatto il ricordo dei cari affetti perduti, in particolare quello del fratello, si ritrovano incastonati nella superficie testuale. Rivelano così la funzione extratestuale che svolsero ai fini dell'esatta evocazione memoriale compiuta dallo scrittore. Inoltre, rimandano alla presenza pulsante di una serie di infratesti autobiografici sottesi alla scrittura. Le immagini del passato, oggetto in esame, riaffiorano nella mente del protagonista unite a oscuri rimorsi che alimentano ulteriormente l'alienante dolore di Gonzalo. Queste paiono delineare una prospettiva che consente di risalire ai motivi di quel profondo malessere intorno al quale si tesse, "opacizzandolo", il discorso narrativo.

Paole chiavi: letteratura e fotografia, La cognizione del dolore, Carlo Emilio Gadda, romanzo italiano del Novecento.

\section{The images of remorse in Gadda's La cognizione del dolore}

\begin{abstract}
The present article analyses a peculiar aspect of Carlo Emilio Gadda's La cognizione del dolore. It is our aim to highlight the link between the photographs belonging to the author and some fragments of the novel. The photographic pictures, particularly his brother's, that keep intact the memory of the lost beloved ones are inserted in the textual surface of the novel. This fact responds to a paratextual function of photographs in order to support the author's remembrance of past events as well as the existence of autobiografical sub-texts that are at work throughout the novel. The past images we refer to are brought to the present by the main character's mind together with obscure remorses that nourish his alienating pain. In brief, they seem to draw a perspective from which it is possible to trace the reasons of the deep unease which is the real objet of a quite inscrutable narrative discourse.
\end{abstract}

${ }^{1}$ Dpto. Traducción e Interpretación, Lenguas Romances, Estudios Semíticos (Área de Filología Italiana). Facultad de Filosofía y Letras. Plaza del Cardenal Salazar s/n. 14003 Córdoba. 
Key words: literature and photography, La cognizione del dolore, Carlo Emilio Gadda, $20^{\text {th }}$ century Italian Novel.

Dal 1938 al 1941 esce a puntate La cognizione del dolore di Carlo Emilio Gadda sulla rivista fiorentina Letteratura, a cui segue a distanza di anni l'edizione in volume del 1963 e la ristampa del $1970^{2}$. Di quest'opera aperta a molteplici interpretazioni, Emilio Manzotti osserva come tracci il cammino di un «graduale, ineluttabile incremento di conoscenza e di dolore» (Manzotti 1996: 208) programmaticamente indicato fin dal titolo. Approfondendo tale indicazione, Dombroski spiega che «l'itinerario di Gadda muove verso l'acquisizione di un oscuro e indecifrabile oggetto, un oggetto dall'origine ignota che il soggetto conoscente custodisce nelle profondità del proprio essere» (Dombroski 2002: 81). L'approssimazione a tale entità, identificata con il malessere morale del protagonista, proietta una "tragica autobiografia", la cui trama però risulta disordinata e incompiuta. Il senso ultimo della vicenda è incomprensibile; non si approda alla piena rivelazione delle «ragioni oscure e vivide» del «male invisibile» e «immedicabile» radicato nell'anima di Gonzalo. Su questo punto, sembra portare chiarezza lo stesso Gadda quando afferma in "Come lavoro» (Paragone, febbraio 1950): «Dovrò, biografando, sorvolare pruni, spini, certe ombre più grevi. Non tutto il dolore è dicibile, non tutto il male e l'orrore» (Gadda 2008a: 427). Nell'operazione conoscitiva sottesa alla scrittura, che per Gadda è strumento di indagine euristica sul reale, vengono liberati «impulsi espressivi» o, come egli meglio precisa, «divergenti impulsi parodistici» (Gadda 2008a: 436). Il tutto è filtrato dalla lente deformante del suo personalissimo stile.

Nel presente articolo si intende raccogliere la sfida ermeneutica lanciata dal romanzo dell'autore milanese, centrando l'analisi del testo su quelle immagini che, per la tensione emotiva che suscitano nel protagonista, possono contribuire a stabilire una prospettiva da cui inquadrare l'indagine sulle cause della sofferenza che lo affligge e lo aliena. Nello specifico, ci si propone di prendere in esame le figure della madre, del padre e del fratello che compongono il nucleo relazionale fondante l'identità del soggetto e rappresentano, nel contempo, gli emblemi di dolore fatto di infinita nostalgia, ma anche di rimorsi e sensi di colpa, che sgorga da trascorsi traumatici cristallizzati nella sua interiorità. L'addentellato con la vita di Gadda è evidente. Si ricordi al riguardo la puntuale esegesi di Contini e lo scambio

${ }^{2}$ Con l'edizione del 1970 si stampa una versione aumentata e definitiva del testo. Vengono aggiunti i due "tratti" finali con cui si mette fine a una complessa e irregolare vicenda editoriale. Sebbene si tratti di frammenti inediti, la nota dell'autore ad essi premessa chiarisce che «i due tratti che seguono appartengono ad uno stadio redazionale più antico [...] Stesi nel ' 41 secondo l'avvertenza dei curatori del volume einaudiano, essi vennero pubblicati solo nel 70 , con la $4^{\mathrm{a}}$ ed. di C.». Le citazioni dal romanzo riportate nel presente articolo sono tratte dall'edizione critica e commentata con un'appendice di frammenti inediti a cura di Emilio Manzotti, Torino, Einaudi (Gli struzzi, 328), 1970 e 1987. 
epistolare tra il critico e il romanziere ${ }^{3}$. D'altro canto, se certo è che il dato autobiografico si ritrova nel fondo del romanzo, tuttavia viene ingarbugliato e complicato dall'esperienza della letteratura. In particolare, riprendendo i preziosi suggerimenti forniti da Terzoli $(1993 ; 2009)$, l'analisi testuale verrà delimitata allo studio del raccordo tra le fotografie di famiglia possedute da Gadda e la costruzione del discorso narrativo, nella fattispecie dell'invenzione letteraria della personalità di Gonzalo. Si prenderà in considerazione, in primo luogo, la funzione svolta dalle fotografie nella storia esterna del romanzo e le eventuali ricadute sulla stesura di alcuni brani, seguendo appunto le indicazioni della studiosa, per poi riflettere sulla valenza che acquistano le riproduzioni fotografiche inserite, nella loro materialità, all'interno della narrazione ${ }^{4}$.

Quando Gadda afferma che «il cosiddetto "uomo normale" è un groppo, o gomitolo o groviglio o garbuglio, di indecifrate (da lui medesimo) nevrosi» (Gadda 2008a: 440), allude all'impossibilità di sciogliere il groviglio di «rapporti físici e metafisici» che fondano la realtà autentica dell'individuo, la cui graduale presa di coscienza accresce il dolore. Ora, quando nella narrazione il punto di vista coincide con la "visione $a b$ interiore" riconoscere, parallelamente all'atto di autoanalisi, una totale assenza di significato alla propria vita «lo hidalgo, forse, era a negare se stesso: rivendicando a sé le ragioni del dolore, la conoscenza e la verità del dolore, nulla rimaneva alla possibilità. Tutto andava esaurito dalla rapina del dolore» (Cognizione: 355). Nonostante ciò, con la figura di Gonzalo, Gadda tenta di circoscrivere e difendere l'area di una storia personale con immagini e situazioni che affermano la consistenza di un'Io che è, al fondo, franto e disgregato. È un compito arduo dato

\footnotetext{
${ }^{3} \mathrm{Nel}$ saggio introduttivo in calce alla prima stampa dell'opera dell'amico (1963), Contini (1989) fa riferimento al ritratto di San Carlo, caro alla madre, distrutto da Gadda in un momento di ira. Dell'episodio si rinviene una trasposizione nel romanzo, anche se qui si tratta del ritratto paterno. In più il critico decifrava il toponimo di Lukones riconoscendovi il reale corrispettivo geografico: Longone al Segrino. Si tratta della località in Lombardia dove il padre di Gadda aveva costruito, con tanti sacrifici e portando la famiglia al bordo del dissesto economico, una casa di campagna. La stessa che appare fedelmente descritta nel romanzo. Va ricordato pure che, nello scambio di missive tra i due, Gadda dava ragione al critico rifiutandosi però di esporsi pubblicamente in tal senso (Gadda 1988: 103-104). Malgrado ciò, solo qualche anno dopo, la foto sbiadita della casa a Longone corredava il volume della quinta edizione del romanzo, quella del 1971.

${ }^{4}$ Le fotografie che corredano i saggi di Terzoli $(1993 ; 2009)$ sono quelle in possesso di Gian Carlo Roscioni, la casa editrice Einaudi e le Edizioni Effigie.

${ }^{5}$ Sul problema del punto di vista, Gadda distingue tra una scrittura narrativa ab interiore, che restituisce "vedendo attraverso la visione del personaggio», e una ab exteriore, in cui l'autorenarratore è l'incaricato di rendere il «lirismo della rappresentazione attraverso i personaggi». La questione va fatta rientrare nella serie di riflessioni sulla forma-romanzo che l'autore sviluppa nei primi anni venti e raccoglie nel Cahier d'études. Gli appunti e le note sono coevi alla stesura della sua prima prova narrativa, Racconto italiano di ignoto del Novecento. Di questi scritti, che risalgono al 1924 e rimangono a lungo inediti, Dante Isella allestisce un'edizione postuma per i tipi di Einaudi nel 1983.
} 
che quegli stessi elementi rimandano a rapporti ambivalenti e paiono indicare obliquamente i motivi di un conflitto interno così profondo da impedire a Gonzalo di immergersi nella pienezza della vita o anche solo di sperare nel conforto della compassione altrui, spingendolo fino alle estreme conseguenze di un pensiero autodistruttivo: la negazione di sé.

Il disegno biografico con cui viene sagomata la personalità finzionale di Gonzalo sembra incardinarsi sulle esperienze individuali vissute dallo stesso Gadda. Va ricordato che, come è noto, il nucleo originario narrativo si configura in concomitanza con la morte della madre dello scrittore, Adele Lehr, deceduta nell'aprile del 1936. L'intensa e dolorosa emozione provocata dal lutto suscitò nel nevrotico autore un grave senso di colpa che divenne un pensiero assillante inscindibile dal ricordo di lei. Scrive al cugino Piero Gadda Conti (27 dicembre 1936) «l'immagine di Lei vecchia e senza aiuti mi ritorna e oltre tutto un indescrivibile rimorso mi prende per i miei scatti, così inutili e così vili. Io ho troppo sofferto e certo non ero padrone di me, ma ciò non toglie che la mia angoscia sia ora grandissima» (Gadda Conti 1974: 42). Il tema dominante, che soggiace alla Cognizione, è proprio il rapporto di amore-odio del protagonista verso la madre. A ciò si aggiunga che Gadda si sbriga a vendere la casa a Longone non appena divenutone l'erede unico, quasi a volersi liberare di un passato dal peso eccessivo, ma contemporaneamente ne fa luogo della memoria rivisitato attraverso la scrittura. È possibile, dunque, supporre che lo sconquassato edificio narrativo abbia fondamenta decisamente traumatiche, legami necessari con antecedenti dolorosi le cui tracce sono parte inscindibile dell'essere del soggetto storico. Il progetto letterario avviato nei primi mesi del 1937 parrebbe rispondere, al pari della vendita della casa di Longone in Brianza ${ }^{6}$, all'urgenza intimamente e tormentosamente sentita dall'autore di liquidare il passato e pacificarsi. Sennonché la scrittura non è mai catarsi per Gadda. Significativamente, in data concomitante alla stesura dei brani che sarebbero confluiti più tardi nell'opera, l'autore confessa in una lettera a Lucia Rodocanachi (16 novembre 1936):

[...] non si liquida tutto un mondo di scarafaggi senza un certo disgusto. Non si rimane soli nel mondo, senza una irredimibile pena. L'immagine di Chi ci ha lasciato ritorna, ritorna, per dirci l'infinità della distanza. La morte di Chi viveva in noi è morte di noi stessi. Molte complicazioni nascono anche dal dolore: che per alcuni è semplicità, per altri uno spaventoso groviglio (Gadda 1983: 60).

\footnotetext{
${ }^{6}$ Gadda tenta, fin dall'estate 1936, di disfarsi di quella villa che era stata in parte la causa, insieme a investimenti agricoli poco avveduti, del tracollo finanziario della famiglia e non da ultimo motivo scatenante di intense dispute tra figlio e madre, la quale, nonostante le evidenti ristrettezze economiche, non si era mai arresa all'idea di venderla. La vendita si rivela più difficile del previsto, ma Gadda, pur concludendo un pessimo affare, sembra trarne un intenso sollievo, quasi un senso di liberazione.
} 
Nella creazione artistica, Gadda complica e ingarbuglia ulteriormente il nodo doloroso che lo affligge, anche perché «L'atto critico, l'atto espressivo non è concepibile per sé, come una emanazione funzionale del bamberottolo io» (Gadda 2008a: 429-430). Che il dato biografico testimoniato nei carteggi dell'autore sia visibile nell'ordito narrativo che si tesse intorno al protagonista della Cognizione è palese, ma certo non risponde, come avverte l'autore stesso, all'idea che la scrittura stringa un legame di mera referenzialità con l'io e le cose.

Tornando al romanzo, va detto che alla proiezione di un mondo "barocco" e "grottesco" realizzata mediante gli ibridismi del maccheronico, si alternano momenti narrativi affidati a modi espressivi tendenti al lirismo nei quali la mente del protagonista insegue le tracce del passato. Queste non vengono ordinatamente ricomposte sulla pagina, ma anzi, disgregate in singoli frammenti, appaiono irregolarmente distribuite quasi a materializzare la memoria della cosa in sé dinnanzi al personaggio e al lettore. È interessante chiedersi se oltre alle frequenti digressioni a cui si lascia andare il protagonista vi siano degli altri dispositivi specifici che, amalgamati nella tramatura della prosa, consentano la manifestazione del passato formando, nel contempo, coaguli di un progetto autobiografico che presenta rilevanti, quanto significativi, parallelismi con l'identità di "ferito", di "dissociato noètico" dell'autore.

Con Terzoli va ipotizzato che lo scrittore possa aver fatto ricorso ai documenti fotografici durante l'atto creativo ai fini di una esatta «mediazione mnemonica per oggetti e persone perdute, e insieme supporto materiale della descrizione» (Gorni 1995). Tale tesi troverebbe una prima giustificazione nell'attenzione scrupolosa prestata dello scrittore ai dati del reale. Inoltre, è risaputo che per anni il milanese custodì gelosamente le riproduzioni fotografiche di cose e persone a lui care. Il desiderio di possesso provato verso quegli oggetti era talmente intenso da spingerlo addirittura a contendere alla madre le ultime missive del fratello. Racconta nella parte finale del Giornale di guerra e di prigionia: «Litigai con la mamma anche per le lettere di Enrico, per le sue memorie» (Gadda 2008b: 864). Se si mettono a confronto alcune istantanee conservate dall'autore e certi luoghi del romanzo in cui vengono presentati la casa, la madre $\mathrm{o}$, con un autoritratto, lo stesso Gonzalo, non è difficile pensare che l'autore le avesse tenute presenti nel momento della creazione. Tra tutti è esemplare la sequenza della madre che vaga sola per la casa. La fisionomia del personaggio, come ha notato Terzoli, sembra rimandare in alcune descrizioni di grande effetto plastico a una rigidità di lineamenti propria del ritratto fotografico in cui il soggetto viene colto nell'immobilità dell'istante. Si legga a modo di esempio il seguente brano:

Il volto, in quelle pause, le si pietrificava nell'angoscia: nessun battito dell'anima era piú possibile: forse ella non era piú persona, ma ombra. Sostava così, nella sala, con pupille cieche ad ogni misericorde ritorno, immobilità scarnita da vecchiezza; per le lunghe falcate del tempo (Gadda 1987: 294). 
D'altro canto la fissità dell'espressione facciale è analoga a quella prodotta dal rigor mortis e, se svela la preoccupazione del personaggio per le condizioni della madre, lascia trasparire altresì la disperata frustrazione dello scrittore al ricordo dell'amato volto non più vivo. Si attua così una sovrapposizione tra il livello letterario dell'enunciazione del personaggio e quello esperito dall'autore, che scrive contemplando le fotografie. In quest'ottica la disposizione prossemica della scena è ulteriomente rivelatrice: la madre, sopraffatta dallo strazio dei ricordi e dallo sfiorire della vita, suggerito metonimicamente dall' «abito di povertà e di vecchiezza» che porta, si trova «davanti ai volti dei ritratti, dove alígeri fatui, sul vuoto, orbiteranno entro il sopravvivente domani» (Gadda 1987: 294-95). La figura della donna è iscritta nell'area formata dai ritratti dei cari defunti suggerendo in tal modo il suo imminente trapasso dalla vita alla morte.

Allo stesso tempo, il brano è rappresentativo dello «slittamento metaforico inerente al documento fotografico, quello tra la persona ritratta e la sua immagine, qui tra i morti e le loro fotografie» (Terzoli 1995) che consente l'affiorare del passato. L'obiettivo è in grado di catturare fattezze e momenti. Li sottrae al fluire degli eventi umani, all'inesorabilità dell'oblio che cancella dal tempo presente dei vivi ogni traccia dei morti dopo la loro dipartita. In questo modo, però, ne viene evocata simultaneamente l'assenza: il fantasma, l'ombra di chi non c'è più viene fissata dalla fotografia diventando "luce opaca". La pagina di Gadda giunge a una densità artistica che travalica il grado più immediato dell'equivalenza tra materiale visuale e linguistico, sulla linea di fusioni che contribuiscono all'intersecarsi disordinato tra vettori di forze tratte dall'irriducibile "molteplicità dei fenomeni" e i piani compositivi dell'opera.

Se, da un punto di vista extratestuale, le fotografie a disposizione dell'autore costituirono un «insieme di frammenti memoriali privilegiati» (Terzoli 1995) che preservavano intatto il ricordo dei cari affetti perduti, queste agiscono nella ricreazione artistica da lui compiuta come matrice autobiografica di immagini che costellano il romanzo. Su tale base, si può congetturare che questi elementi testuali sono in grado di rimandare a infratesti attivi nella proiezione della figura del protagonista. A questo proposito è interessante soffermarsi sulle condizioni di significato che il documento fotografico stabilisce con la narrazione della «vicenda oscillante d'uno spirito fugitivo e aleatorio» (Gadda 2008a: 427). Innanzi tutto si osservi che le fotografie usate da Gadda e di cui egli offre una trasposizione parziale nel romanzo coincidono con alcune di quelle «vane immagini» (Gadda 1987: 355) che Gonzalo richiama alla mente nel tentativo disperato di affermare l'unità della propria identità scissa. L'autore ricorre alle componenti tipiche della psicoanalisi quali la proprietà (la villa) e i legami parentali ${ }^{7}$ (la madre, il padre, il fratello) (Manzotti 1987: xlii-xliii). Nell'operazione artistica, tuttavia, quelle cose e

${ }^{7}$ A suggello di questa dinamica psicologica, va segnalato un interessante elemento linguistico. Gonzalo, prima di essere chiamato con il nome proprio, è nominato come "figlio", "discendente hidalgo" e simili. 
persone si presentano con il loro carico di tensioni irrisolte, non pacificate, di sensi di colpa e di rimorsi che non fanno altro se non esacerbare il dolore di Gonzalo inducendolo alla negazione del proprio essere. Questi elementi sembrano delineare una prospettiva in cui inquadrare i motivi scatenanti e/o integranti tale malessere, vero oggetto della narrazione, la cui causa rimane in ultima istanza innominata, così come risulta indecifrabile il significato dell'intero romanzo.

Riprendendo il filo del discorso, per quanto riguarda la presenza nel discorso narrativo di alcune fotografie richiamate nella loro concreta fisicità di oggetti, è interessante rifarsi ai concetti di "indice" e di "icona" formulati da Charles Sanders Peirce. Va premesso, infatti, che il documento fotografico può assumere una funzione indicativa, in quanto indice di un dato reale, e una funzione analogica basata sulla sua somiglianza, in quanto icona (Peirce 1980: 156-171). Le immagini fotografiche che fanno parte integrante del testo non sostituiscono di certo l'elemento concreto ma vengono fatte agire secondo la loro potenza referenziale: fondono in sé icona e indice secondo le intenzioni di chi le concepisce come calco di un ombra, dotato di un pulsante contenuto emotivo. D'altro canto, l'interazione tra l'oggetto iconico e il soggetto doveva essere ricca di suggestioni per il Gadda romanziere e filosofo, in quanto concretizza visivamente lo stabilirsi, e dunque l'aggrovigliarsi, di rapporti fisici e metafisici con la conseguente ingerenza di elementi esterni nello spazio intimo e percettivo dell'Io.

Incastonate nella superficie della narrazione, si ritrovano i ritratti fotografici del padre e del fratello del protagonista. La loro presenza non è da attribuire ad un fine documentaristico, quanto piuttosto all'intenzione di restituire la presenza simbolica dei familiari defunti nella vita di Gonzalo. In quest'ottica le effigie del padre e del fratello fungono da correlativi oggettivi del dolore provocato dalla perdita dei cari e, nel contempo, da emblemi delle complicazioni connesse al lutto che «per alcuni è semplicità, per altri uno spaventoso groviglio» come l'autore dichiara nella già ricordata lettera del 1937 a Lucia Rodocanachi.

$\mathrm{Al}$ ritratto del padre si fa più volte riferimento nel corso della narrazione. Si tratta del quadro appeso in salotto, nella grande sala adiacente alla terrazza, accanto a quella di altre fotografie familiari, tra cui presumibilmente quella del figlio scomparso in guerra. Nel loro insieme costituiscono, si è già visto, i muti interlocutori della madre sofferente (Gadda 1987: 294-295).

L'altro ritratto del padre, quello che si trova nella casa di città, è invece al centro di una vicenda che si mostrerà particolarmente significativa per la ricostruzione di un livello di senso della narrazione. La vicenda è introdotta in due momenti cruciali del romanzo: precede la presentazione del protagonista all'inizio ed è inserita durante un lungo atto di autoanalisi verso la fine. La storia è prima anticipata dalla Battistina (Gadda 1987: 126), la quale la riferisce al medico che si sta recando da Gonzalo. Il dottore, a sua volta, rievoca il racconto fra sé e sé, arricchendolo di particolari raccolti da altre voci, in un passaggio in cui domina l'impiego del discorso indiretto libero (Gadda 1987: 132-133). Pìu avanti è poi evocato dal narratore con punto di vista interno al personaggio (Gadda 1987: 374-375). L'episodio ripetuto è quello del quadro del padre gettato a terra e calpestato da 
Gonzalo durante un violento rimprovero alla madre colpevole di aver acquistato un orologio da regalare al figlio appena laureato. La rappresentazione dell'oltraggio alla memoria del progenitore convoglia, soprattutto nella confessione del figlio, un acuto senso di rimorso, di dolore. Allo stesso tempo, sembra alludere a qualcosa di ben più indicibile, il patricidio. Difatti, se messo in correlazione al desiderio di possesso totale espresso nei confronti della madre, il gesto di Gonzalo può essere spiegato nei termini psicoanalitici del complesso edipico. Tuttavia il triangolo Gonzalo-Madre-Padre non regge, come suggerisce Contini (1989). Basti solo pensare che la conclusione della vicenda, con la morte della madre, sembra addirittura ribaltare tale interpretazione e suggerire piuttosto il motivo del matricidio. La storia su cui si insiste costituirebbe, dunque, uno degli indizi sulla cui base il protagonista viene sospettato quale autore del delitto.

Ai fini della presente analisi, occorre soffermarsi sul brano con cui termina il romanzo. Nella scena finale, infatti, si rinviene un'altra fotografia: dalla camera da letto, dove giace la madre moribonda, è perfettamente visibile allo sguardo indiscreto di chi è accorso in aiuto alla "Signora". Si legge «sul tavolo un libro aperto, una fotografia del fratello di lui, ragazzo dal volto sorridente, dopo tant'anni!: con una mano sul manubrio della mitragliatrice: era visibile, in parte, la struttura del velivolo» (Gadda 1987: 463). L'istantanea si trova nella stessa stanza in cui, all'inizio, il medico aveva visitato Gonzalo ed era avvenuta la diagnosi della malattia morale che lo affligge. Eppure, ritornando a quella sequenza, il ritratto non è compreso nel resoconto particolareggiato degli oggetti (i due letti, i copriletti bianchi, le finestre, il dagherrotipo di Garibaldi) che riempiono lo spazio della camera. Retrospettivamente tale omissione assume un'incontrovertibile rilevanza.

A questo riguardo, Federica Pedriali (1997) propone una diversa lettura del dolore di Gonzalo e del senso di tutto il romanzo, partendo dall'idea che il dipanarsi della narrazione risponda alla dinamica di un complesso edipico in cui «il terzo elemento del triangolo è Enrico, non il padre di Gadda» (Pedriali 1997: 137). Il figlio sopravvissuto contende, nel profondo, al fratello morto l'amore materno. Il senso di esclusione e di invidia scatena pensieri ignominiosi che provocano irredimibili «lacerazioni del sentire» (Gadda 2008a: 429). Di conseguenza, Gonzalo viene «condannato all'oscurità dalla perfidia della madre [che a lui preferisce il fratello], offuscato dal sorriso acceccante del fratello, senza nessuno che riconosca la sua superiorità morale, né la madre né la plebaglia che ella protegge» (Pedriali 1997: 150).

Se, accogliendo la tesi della studiosa, la causa ultima e irreparabile del "male oscuro" del protagonista va ricercata nel rapporto con il fratello, la foto parebbe svolgere un ruolo di primaria importanza. Ad ogni modo, colpisce l'esigua quantità dei riscontri, ridotti a pochi dettagli quasi scollegati e marginali rispetto all'ordito narrativo. Si fa allusione veloce al fratello ricordandone i ritratti conservati dalla madre nel «vecchio secrétaire di noce ch'ella non riusciva più a disserrare» (Gadda 1987: 293). È pure probabile che uno dei ritratti che si trovano nella "grande sala" vicino a quello del padre sia il suo. Tuttavia l'identità del individuo in essi immortalato non è messa a fuoco. Soltanto nella scena finale, come si diceva, essa sarà indicata chiaramente con un effetto di svelamento ritardato. 
Ricollegandoci a un più che plausibile utilizzo delle fotografie da parte di Gadda nel momento della creazione artistica, va spiegato che, benché scarse e poco decifrabili siano le immagini del fratello confluite nel racconto, è possibile ipotizzare che la figura veicolata dal ricordo della madre e da quello di Gonzalo sia sagomata sulla base delle istantanee gelosamente serbate dall'autore. Le descrizioni del fratello del protagonista presentano elementi che fanno pensare a un ritratto fotografico. E ancora, come per la madre, si ripete il traslato tra riproduzione fotografica e morte:

Con occhi lucidissimi, aperti. Aperti, fermi. Nello stupore del sogno senza più risposte. La favola. Era chiara, ora, splendida, interminata, come nel libro del bimbo. Due fili di sangue gli scendevano dalle narici sui labbri, semiaperti: dischiusi alla verità impronunciabile. (Gadda 1987: 415)

Le fotografie che perpetuano il ricordo del giovane aviatore sono le stesse che, contemplate dall'autore molti anni prima, lo portano ad essere consapevole della realtà della perdita. In questo senso, della funzione svolta da questi documenti si riscontra una significativa testimonianza nelle prime prose di Gadda, quelle nate sull'onda dello sgomento prodotto dall'annuncio della morte del fratello. Di ritorno dalla prigionia, il 13 gennaio 1919, Gadda apprende il tragico destino toccato in sorte al congiunto. In data 31 dicembre 1919, alla fine del suo Giornale di guerra e di prigionia, scrive «Riguardo e penso i ritratti del nostro Enrico adorato, e nella desolazione vorrei avere una fede, la certezza di rivederlo dove che sia. Ma non lo vedrò mai: il suo sorriso è cosa del passato indimenticabile» (Gadda 2008b: 867). Si confronti allora il brano che chiude il Giornale con quello che conclude il testo intitolato «Impossibilità di un diario di guerra» (L'Ambrosiano, 7 dicembre 1931) ${ }^{8}$ : «Alcuni avevano una catenella d'oro al polso e morirono come fanciulli, sognando il Natale: avevano nel viso una luce, un sorriso: e l'angoscia mi riconduce pei vani sentieri della memoria, ma tutto tace, intorno, e tutto si oscura» (Gadda 2007: 144). Ritorna lo stesso identico motivo nella Cognizione «il figlio che le aveva sorriso, brevi primavere!» (Gadda 1987: 256); «Chi si amava è nella terra... Era nel suo viso una luce... un sorriso» (Gadda 1987: 194); «il fratello, sorriso lontano! Chiusone in sé il nome, la disperata memoria» (Gadda 1987: 291). Il tema del sorriso costituisce una tessera di una tale insistenza da diventare un tratto identificativo, per antonomasia, del giovane. Considerando dunque che la maggior parte delle brevi descrizioni e dei veloci riferimenti al fratello sono esemplati sull'immagine di un giovane arruolato in aviazione deceduto tragicamente in guerra, se si pretende indagare il contenuto emotivo nascosto che affiora insieme a tali ricordi anche nella scrittura della Cognizione, non si può prescindere né dalle pagine diaristiche del Giornale di guerra e di prigionia né dalle prose post-belliche unite ne Il castello di Udine (1934).

\footnotetext{
${ }^{8}$ Poi ripubblicato nel volume Il castello di Udine (1934).
} 
Nel Giornale si ritrovano numerose tracce dell'intensa angoscia vissuta dall'autore a causa dell'incertezza sulla sorte dei congiunti. Tuttavia, assieme alla nostalgia e al timore per il fratello, spiccano delle zone d'ombra e dei silenzi che, soprattutto dal confronto di queste cronache belliche con i testi raccolti in Castello, lasciano scorgere un sentimento di invidia nei confronti del fratello (Gorni 1995). $\mathrm{Ne}$ è prova un brano di «Compagni di prigionia» in cui si rende, mediante la simultaneità del reale, la situazione di chi fuori dal campo di prigionia combatteva e chi dentro invece pativa l'umiliazione di essere stato catturato «la sera scendeva, nuvole basse trasvolavano sopra i fari del campo, rotonde e livide: quasi a lacerarsi nel filo spinato [...] Le finestrette delle baracche si illuminavano, e noi camminare e camminare» (Gadda 2007: 165). È utile ricordare che Gadda, fatto prigioniero dai tedeschi il 25 ottobre del 1917 durante la disfatta di Caporetto, visse la reclusione come un'onta, uno scacco esistenziale, una vergogna di cui soffre ancor di più dopo il congedo, al pari di Gonzalo è «reduce senza endecasillabi» (Gadda 1987: 288). Il suo desiderio di distinzione nel compimento del proprio dovere sembra portarlo a covare un senso di astio verso il fratello aviatore. Crede che si stia coprendo di gloria nella battaglia, mentre lui è condannato all' «inanità morale della prigionia: dai regni fulgidi, dopo i fulgidi atti del cosciente volere, ero stato travolto verso la riva dell'inutilità» (Gadda 2007: 171). È possibile ipotizzare, con Gorni (1995), che Gadda si sia reso conto di aver nutrito questi sentimenti durante l'elaborazione del lutto. Si tratta di pensieri che, seppur maturati nell'alterazione causata dall'esperienza della guerra e del campo di lavoro, non potevano non ripresentarsi all'annuncio della morte di Enrico, per poi trasformarsi in una ragione di tormento e di rimorso indimenticabili. A sostegno di tale suggestione, e del radicarsi quasi tumorale di siffatto conflitto nell'anima dello scrittore milanese, va ricordata con Roscioni l'espressione sgomenta che trasfigurò il volto di Gadda alla vista del quadro caravaggesco, Caino e Abele, posto in copertina all'edizione del Giornale del 1965 (Terzoli 1995). Se l'editore intendeva con quel motivo figurativo condannare tutte le guerre in quanto fratricide, a Gadda dovette senz'altro toccare qualche fibra sensibile.

Ritornando allora alla Cognizione, la fotografia che ritrae il fratello a fianco dell'aeroplano militare, richiamata esplicitamente nelle sequenze drammatiche del finale, rappresenterebbe il segno indelebile di una ferita che ulcera Gonzalo e replica quella di Gadda. L'immagine della memoria agisce sulla scrittura e nel racconto sulla scorta di un occulto e intricato palinsesto psicologico che acquista vividezza solo alla fine. Sebbene il ritratto del caduto si palesi in modo diretto nell'ultimo tratto, il nono, il peso dell'assenza del giovane scomparso e il permanere a distanza di anni del lutto gravano su tutto il romanzo. Non è un caso se la visita medica, a cui si sottopone il protagonista all'inizio, nel terzo tratto, venga collocata nel luogo del ricordo e del dolore, vale a dire la stanza che una volta era stata occupata dai due fratelli. Dopo la morte il fratello diventa una presenza simbolica e di ciò gli oggetti diventano eloquenti testimoni «il figlio si liberò della giacca, si sdraiò sul letto più interno, il suo: di coltre bianchissima, come l'altro, di pesante noce» (Gadda 1987: 139). Di tutti, però, si tace del più emblematico in tale 
senso, il ritratto fotografico. Omesso da questa prima descrizione della camera da letto di Gonzalo, è soltanto nell'epilogo del romanzo, nel momento in cui è scoperto l'orrendo crimine perpetuato da ignoti, che viene esposto agli sguardi degli intrusi. Sulla scrivania di Gonzalo, accanto agli amati libri, c'è la foto del fratello in guerra.

Perciò, tornando alla prima descrizione della camera, colpisce tale mancanza la quale getta, nel contempo, una luce diversa su altri elementi, svelando stratificazioni di significato grazie proprio alla chiave interpretativa offerta dal rapporto con il fratello. Si pensi, ad esempio, all'eccessiva attenzione prestata al dagherrotipo di Garibaldi («generale Pastrufacio»). La sovrabbondanza di particolari e l'insistenza sulle qualità di «nobilità», di «generosità maschia», di «vigore» che emana dal «mezzo busto nella penombra» (Gadda 1987: 141) contrastano con la debolezza del finto malato. L'esame medico avviene proprio sotto lo sguardo corrucciato del prode quasi a ridicolizzare il protagonista, tanto più che le cause del male che lo emarginano dal vivere sociale non sono fisiche. Di conseguenza risultano incomprensibili, e perfino oggetto di scherno, non solo per la gente comune ma anche per lo stesso dottore. La presenza dell'eroe del Risorgimento italiano non è casuale anche per un'altra ragione. È elemento cifrato che fa riferimento, per apposizione, al fratello eroicamente morto in difesa della patria. Nell'incipit della scena, la pagina acquista visività pittorica grazie ai giochi cromatici di bianco e nero polarizzati dal motivo iterato del legno del noce e dall'immagine della «coltre bianchissima». Inoltre, questi assumono un valore metaforico dal momento che mutano lo spazio che denotano suggerendo uno slittamento di senso: dalla stanza al feretro (quello del fratello). A chiudere il cerchio è altresì l'identificazione operata tra Gonzalo e il cadavere del congiunto «su quel candore conventuale il lungo corpo e la eminenza del ventre diedero una figurazione di ingegnere-capo decentemente defunto» (Gadda 1987: 140). Il tormento provato per il fratello morto e commisto a un doloroso senso di colpa si può inferire solo tenendo presente il brano finale. E di ciò è particolarmente rivelatrice l'immagine del giovane soldato.

Riprendendo la tesi di Pedriali, è utile osservare che prima dello pseudosvelamento della conclusione, lo strazio lacerante provocato dalla morte in guerra del fratello è manifestato in tutta la sua insondabile profondità solo attraverso il fluire dei ricordi della madre, vale a dire dal suo punto di vista e mediante il reiterare quasi ossessivo dell'esperienza del mancato ritorno del figlio. Nel dispiegarsi del passato lungo il filo della memoria di questo personaggio, il narratore spesso si inserisce per mettere in ridicolo Gonzalo proprio sul valore dimostrato in guerra dall'uno e non dall'altro. Il discorso acquista allora un tono paradossale e di scherno in considerazioni del tipo «a Gonzalo, no, no! Non erano stati tributati i funebri onori delle ombre» (Gadda 1987: 273), oppure «Oh! Il "suo" Gonzalo! Era troppo evidente che l'arsenale della gloria aveva rifiutato di prenderlo in carico. Plauto, in lui, non troverebbe il suo personaggio, forse Molière» (Gadda 1987: 289). Il dolore inconsolabile della madre per il figlio morto sembra spingere Gonzalo a condividere la sua visione. Pare percepire il fratello quale figlio ideale da lei proiettato e, di conseguenza, a giudicare se stesso come «prova difettiva di 
natura, un fallito sperimento delle viscere» (Gadda 1987: 271-272). Parimenti, l'immagine della madre chiusa nel lutto e nella venerazione della memoria del caduto alimentano nel protagonista un sordo livore e un sentimento di ira contenuta. Quel senso di indicibile invidia verso l'eroe dei cieli, "nodo" insieme personale e storico che, documentato negli appunti del Giornale, è ripreso nella Cognizione, si lega qui ad una ben più indicibile empietà:

Ma, tutto, tutto, è bene che si soppesi. Il figlio pareva aver dimenticato al di là d'ogni immagine lo strazio di quegli anni, la incenerita giovinezza. Il suo rancore veniva da lontananza piú tetra, come se fra lui e la mamma ci fosse qualcosa di irreparabile, di piú atroce d'ogni guerra e d'ogni spaventosa morte (Gadda 1987: 317).

Il figlio rimasto in vita non può contendere al fratello l'intensità dell'amore materno, come da vivo non aveva potuto competere con il suo eroismo. In questo groviglio, trapela il trauma del sopravvissuto che dovette segnare intimamente l'autore. L'esser tornato dal fronte è sentito come una colpa aggravata dalla vergogna provata per la gelosia sentita verso Enrico. Un sentimento maturato quando questi era ancora vivo e destinato a rimanere tale anche dopo la sua scomparsa: con la morte era stato posto sul piedistallo dell'eroismo e quale martire veniva rimpianto e adorato da tutti, in primis dalla madre. Gonzalo-Gadda non può aspirare né a sostituirsi al fratello, né a ricevere le lodi, l'ammirazione e l'amore che avrebbe meritato invece lui. Gonzalo è costretto a permanere, senza alcuna opportunità di riscatto e di risarcimento, nella zona d'ombra della vita roso da rimorsi indicibili. In questo senso, la fotografia del fratello posta nell'epilogo della vicenda rivela la contrapposizione dicotomica tra i due individui. La luce nel viso del primo è segno dell'intesa ed eroica immersione nella pienezza della vita negata invece al secondo a causa della sua stessa ricerca «delle cause, origini, forma prima, sviluppo» delle proprie nevrosi di uomo «anormale» (Gadda 2008a: 441) ${ }^{9}$.

Per concludere, va detto brevemente che nel romanzo in questione non solo si occulta la sofferenza, ma anche i conflitti interni che essa abbia potuto provocare in Gadda e che si possono rintracciare in tutta la sua opera sotto la parvenza di immagini e situazioni ricorrenti. Nel progetto autobiografico di Gadda la luce opaca dei morti, quella riflessa da "Chi" mai piú ritorna, restituisce alla coscienza del protagonista le ambivalenze inerenti a quei rapporti, fonte di ricordi poco onorevoli e di rimorsi che indicano indirettamente le cause da cui si sviluppa il conflitto perenne di Gonzalo. Ne deriva che nella compagine dell'opera possono essere

\footnotetext{
${ }^{9}$ Va anche ricordato che il giovane Gadda, ancora studente al Politecnico di Milano, era stato infiammato e affascinato dall'oratoria nazionalistica del D'Annunzio interventista. Non è azzardato immaginare che al di sotto di questa fotografia si nasconda il rimando a un particolare referente extratestuale, vale a dire le celebri istantanee scattate al Vate accanto ai velivoli di guerra durante le sue eroiche azioni sul fronte austriaco, e non solo. Nel 1910 lo scrittore abruzzese pubblica Forse che sì, forse che no, in cui rappresenta, con l'automobile e l'aeroplano, le forme eroiche della moderna civiltà.
} 
rintracciate corrispondenze profonde tra dati biografici e nuclei tematici che non di rado l'elemento fotografico, inglobato nella scrittura, rinsalda e suggerisce.

\section{RIFERIMENTI BIBLIOGRAFICI}

ABRUZZESE, Alberto; GRASSI, Carlo (1986): «La fotografia» in A. Asor Rosa (ed.) Letteratura italiana. Storia e Geografia. III. L'età contemporanea, Torino, Einaudi, pp. 1177-1222.

BERTONE, Manuela (1997): «Murderous Desires: Gaddian Matricides from Novella seconda to La cognizione del dolore» in M. Bertone, R. Dombroski (eds.), Carlo Emilio Gadda. Contemporary Perspectives, Toronto, University of Toronto Press, pp. 11-131.

CONTINI, Gianfranco (1989): «Introduzione» in Quarant'anni di amicizia. Scritti su Carlo Emilio Gadda (1934-1988), Torino, Einaudi, pp. 15-35 [C. E. Gadda (1963): La cognizione del dolore, Torino, Einaudi, pp. 5-28].

DOMBROSKI, Robert S. (2002): «Un'etica barocca», Gadda e il barocco, Torino, Bollati Boringhieri, pp. 81-102.

GADDA, Carlo Emilio (1983): Lettere a una gentile signora, a cura di G. Marcenaro, Milano, Adelphi.

GADDA, Carlo Emilio (1987): La cognizione del dolore, Torino, Einaudi.

GADDA, Carlo Emilio (1988): Lettere a Gianfranco Contini a cura del destinatario. 1934-1967, Milano, Garzanti

GADDA, Carlo Emilio (2007 [1988]): Romanzi e racconti I, a cura di R. Rodondi, G. Lucchini, E. Manzotti, edizione diretta da D. Isella, Milano, Garzanti.

GADDA, Carlo Emilio (2008a [1991]): «Come lavoro», Saggi, Giornali, Favole e altri scritti I, a cura di L. Orlando, C. Martignoni, C. Segre; edizione diretta da D. Isella, Milano, Garzanti, pp. 427-443.

GADDA, Carlo Emilio (2008b [1992]): «Giornale di guerra e di prigionia», Saggi, Giornali, Favole e altri scritti II, a cura di C. Vela, G. Gaspari, G. Pinotti, F. Gavazzeni, D. Isella e M.A. Terzoli, edizione diretta da D. Isella, Milano, Garzanti, pp. 431-867.

GAdDA ConTI, Piero (1974): Le confessioni di Carlo Emilio Gadda, Milano, Pan.

GORNI, Guglielmo (1995): «Gadda, o il testamento del capitano», in M. A. Terzoli (ed.), Le lingue di Gadda, Roma, Salerno Editore, pp. 149-178. Si cita dal testo pubblicato in The Edinburgh Journal of Gadda Sudies Archives $<$ http://www.gadda.ed.ac.uk/Pages/resources/archive/war_writings/gornicapitano. php $>$ [consultato il 6 maggio 2012]

MANZOTTI, Emilio (1996): «La cognizione del dolore di Carlo Emilio Gadda», in A. Asor Rosa (ed.), Letteratura italiana. Le opere, IV.2: La ricerca letteraria, Torino, Einaudi, pp. 201-337.

MANZOTTI, Emilio (1987): «Introduzione» in C. E. Gadda, La cognizione del dolore, Torino, Einaudi, pp. vi-li. 
MILESCHI, Christophe (2003): «"La guerra è cozzo di energie spirituali”: estetica ed estetizzazione della guerra in Carlo Emilio Gadda», Bollettino '900, 9 (1), $<$ http://www.boll900.it/2003-i/Mileschi.html> [consultato il 14 aprile 2012]

PEDRIALI, Federica (1997): «The mark of Cain: Mourning and Dissimulation in the Works of Carlo Emilio Gadda», in M. Bertone, R. Dombroski (eds.), Carlo Emilio Gadda. Contemporary Perspectives, Toronto, University of Toronto, pp. $132-58$

PEDRIALI, Federica (2007): «Cain and other symmetries», The Edinburgh Journal of Gadda Sudies, 6. <http://www.gadda.ed.ac.uk/Pages/journal/ monographs/ pedriali/pedricain2.php> [consultato il 12 aprile 2012]

PEIRCE, Charles Sanders (1980): Semiotica. I fondamenti della semiotica comunicativa, Torino, Einaudi, pp. 156-71.

RAIMONDI, Ezio (2003): Barocco moderno. Roberto Longhi e Carlo Emilio Gadda, Milano, Mondadori.

Terzoli, Maria Antonietta (1993): La casa della «Cognizione». Immagini della memoria gaddiana, Milano, Effigie.

TERZOLI, Maria Antonietta (1995): «Le immagini della memoria» in M. A. Terzoli (ed.), Le lingue di Gadda, Roma, Salerno Editore, pp. 225-246 [si cita dal testo pubblicato sulla rivista online The Edinburgh Journal of Gadda Sudies (2000/0). $<$ http://www.gadda.ed.ac.uk/Pages/journal/issue0/articles/terzolimemoria.php> [consultato il 12 aprile 2012]

TERZOLI, Maria Antonietta (2009): Alle sponde del tempo consunto. Carlo Emilio Gadda dalle poesie di guerra al «Pasticciaccio», Milano, Effigie. 